\title{
A DERIVATION OF MODIFIED NEWTONIAN DYNAMICS
}

\author{
SASCHA TRIPpE \\ Department of Physics and Astronomy, Seoul National University, Seoul 151-742, Korea \\ E-mail : trippe@astro.snu.ac.kr \\ (Received February 14, 2013; Revised March 20, 2013; Accepted March 29, 2013)
}

\begin{abstract}
Modified Newtonian Dynamics (MOND) is a possible solution for the missing mass problem in galactic dynamics; its predictions are in good agreement with observations in the limit of weak accelerations. However, MOND does not derive from a physical mechanism and does not make predictions on the transitional regime from Newtonian to modified dynamics; rather, empirical transition functions have to be constructed from the boundary conditions and comparisons with observations. I compare the formalism of classical MOND to the scaling law derived from a toy model of gravity based on virtual massive gravitons (the "graviton picture") which I proposed recently. I conclude that MOND naturally derives from the "graviton picture" at least for the case of non-relativistic, highly symmetric dynamical systems. This suggests that - to first order - the "graviton picture" indeed provides a valid candidate for the physical mechanism behind MOND and gravity on galactic scales in general.
\end{abstract}

Key words : Gravitation - Galaxies: kinematics and dynamics

\section{INTRODUCTION}

\begin{abstract}
"It is worth remembering that all of the discussion [on dark matter] so far has been based on the premise that Newtonian gravity and general relativity are correct on large scales. In fact, there is little or no direct evidence that conventional theories of gravity are correct on scales much larger than a parsec or so."

— Binney \& Tremaine (1987), Ch. 10.4, p. 635
\end{abstract}

Since the seminal works by Zwicky (1933) and Rubin et al. (1980), it has become evident (e.g., Binney \& Tremaine 1987; Sanders 1990) that the dynamical masses of galaxies and galaxy clusters exceed their luminous (baryonic) masses by up to one order of magnitude - the well-known missing mass problem. A possible solution is provided by Modified Newtonian Dynamics (MOND) which postulates a modification of the classical Newtonian laws of inertia and/or gravity in the limit of weak accelerations (Milgrom 1983a,b,c; Bekenstein \& Milgrom 1984; Sanders \& McGaugh 2002; Bekenstein 2006; Ferreira \& Starkman 2009; Famaey \& McGaugh 2012). Assuming a test particle on a circular orbit around a baryonic mass $M_{0}$ at distance $r$ with circular speed* $v_{\mathrm{c}}$, MOND relates the centripetal acceleration $a_{\mathrm{c}}=v_{\mathrm{c}}^{2} / r$ and the Newtonian acceleration $g_{\mathrm{N}}=G M_{0} / r^{2}$, with $G$ being Newton's constant, as

$$
\frac{g_{\mathrm{N}}}{a_{\mathrm{c}}}=\mu(x)
$$

\footnotetext{
*For simplicity, I only regard absolute values of velocities and accelerations; the orientations are evident from the assumed geometry.
}

where $x=a_{\mathrm{c}} / a_{\mathrm{M}}, a_{\mathrm{M}} \approx 10^{-10} \mathrm{~m} \mathrm{~s}^{-2}$ is Milgrom's constant, and $\mu(x)$ is a transition function with the asymptotic behavior $\mu(x) \rightarrow 1$ for $x \gg 1$ and $\mu(x) \rightarrow x$ for $x \ll 1$. The first limiting case corresponds to standard Newtonian dynamics. The second limiting case leads to $v_{\mathrm{c}}{ }^{4} \approx G M_{0} a_{\mathrm{M}}=$ const.; this explains the asymptotic flattening of galactic rotation curves, the Tully-Fisher/Faber-Jackson relations, and (via division by $r^{2}$ ) the surface brightness-acceleration relation of galaxies (see, e.g., Famaey \& McGaugh 2012 for a recent review).

Despite its success, MOND is obviously incomplete. Firstly, it does not derive from a physical mechanism a priori. Second, even though it provides the correct limiting cases by construction, MOND does not provide $\mu(x)$ itself and makes no prediction on the transitional regime from Newtonian to modified dynamics. This is especially unfortunate given the fact that the transitional regime has been explored by observations: the empirical mass discrepancy-acceleration (MDA) relation (McGaugh 2004) shows that the ratio $M_{\text {tot }} / M_{0}$ (the mass discrepancy) is a characteristic function of the accelerations $a_{\mathrm{c}}$ and $g_{\mathrm{N}}$; here $M_{\text {tot }}$ is the total dynamical mass given by $a_{\mathrm{c}}=G M_{\mathrm{tot}} / r^{2}$. If a prediction for $\mu(x)$ was available, it could be tested by comparison to the empirical MDA relation in a straightforward manner.

Recently, I proposed a scheme for gravitational interaction on galactic scales (the "graviton picture") which is based on the ad-hoc assumption that gravity is mediated by virtual massive gravitons that obey certain reasonable rules of interaction (Trippe 2013). The "graviton picture" predicts a theoretical MDA re- 
lation which is in good agreement with observations; it comprises expressions for limiting cases that agree with those of MOND (and likewise agree with observations). The present work follows up on, and amends, Trippe (2013). I realized only after publication of Trippe (2013) an additional consequence of my toy model of gravity introduced there: A comparison of classical MOND and "graviton picture" shows that MOND naturally derives from the "graviton picture" at least for the case of non-relativistic, highly symmetric dynamical systems. This comparison is the subject of the present work.

\section{ANALYSIS}

\subsection{Transition Functions in MOND}

Eq. 1 provides the defining properties of MOND; the transition function $\mu(x)$ is constrained by (1) limiting cases that have to be consistent with observations (2) the condition that $x \mu(x)$ increases monotonically with $x$ (Famaey \& McGaugh 2012). These conditions are fulfilled (e.g., Milgrom 1983a; Famaey \& Binney 2005; Famaey \& McGaugh 2012) by the set of functions

$$
\mu_{n}(x)=\frac{x}{\left(1+x^{n}\right)^{1 / n}} ; \quad n=1,2,3, \ldots .
$$

Alternatively, one may re-write Eq. 1 as

$$
\frac{a_{\mathrm{c}}}{g_{\mathrm{N}}}=\nu(y)
$$

where $y=g_{\mathrm{N}} / a_{\mathrm{M}} ; \nu(y) \rightarrow 1$ for $y \gg 1$ and $\nu(y) \rightarrow y^{-1 / 2}$ for $y \ll 1$. For reasons analogous to those for the case of $\mu(x)$, a set of valid transition functions is given by (Famaey \& McGaugh 2012)

$$
\nu_{n}(y)=\left\{\frac{1}{2}\left[1+\left(1+\frac{4}{y^{n}}\right)^{1 / 2}\right]\right\}^{1 / n} .
$$

Comparison to the empirical MDA relation suggests $n=1$ or $n=2$ (Kroupa 2012). Nevertheless, none of these scaling relations follows from first principles: technically, arbitrary alternative transition functions can be constructed from comparison to the data.

\subsection{The "Graviton Picture"}

The "graviton picture" (Trippe 2013) employs the ad-hoc assumption that gravity is mediated by virtual gravitons with non-zero mass that obey certain reasonable rules of interaction. This leads to the formation of a "graviton halo" with a mass density profile $\rho \propto r^{-2}$ around a baryonic source mass $M_{0}$. The total dynamical mass $M_{\text {tot }}$ scales with centripetal acceleration $a_{\mathrm{c}}$, providing a theoretical MDA relation

$$
\frac{M_{\mathrm{tot}}}{M_{0}}=1+8 \pi \frac{a_{0}}{a_{\mathrm{c}}}
$$

where $a_{0}$ is a constant of the dimension of an acceleration. The limiting case $a_{\mathrm{c}} \gg 8 \pi a_{0}$ corresponds to the usual Newtonian dynamics. The limiting case $a_{\mathrm{c}} \ll 8 \pi a_{0}$ leads to $v_{\mathrm{c}}{ }^{4} \approx 8 \pi G M_{0} a_{0}=$ const.; from comparison to the corresponding MOND result we find $a_{\mathrm{M}} \equiv 8 \pi a_{0}$.

In contrast to MOND, the "graviton picture" comprises a scaling law for the transitional regime from Newtonian to modified dynamics (Eq. 5) a priori. In analogy to Eq. 1 we can define a transition function

$$
\xi(x)=\frac{g_{\mathrm{N}}}{a_{\mathrm{c}}}=\frac{M_{0}}{M_{\mathrm{tot}}}=\left[1+\frac{1}{x}\right]^{-1}
$$

with $x=a_{\mathrm{c}} / a_{\mathrm{M}}$ as before. This results in

$$
\xi(x)=\frac{x}{1+x}=\mu_{1}(x) .
$$

As we see, the transition function $\xi(x)$ provided by the "graviton picture" is identical to the MOND transition function $\mu_{n}(x)$ for $n=1$.

Furthermore, we can define an inverse transition function in analogy to Eq. 3 as

$$
\zeta=\frac{M_{\mathrm{tot}}}{M_{0}}=\frac{a_{\mathrm{c}}}{g_{\mathrm{N}}} \longrightarrow y=\frac{g_{\mathrm{N}}}{a_{\mathrm{M}}}=\frac{x}{\zeta} .
$$

Via Eq. 5 this leads to the quadratic equation

$$
\zeta^{2}-\zeta-\frac{1}{y}=0
$$

Solving this expression for $\zeta$ and ignoring the unphysical negative root, we find

$$
\zeta(y)=\frac{1}{2}\left[1+\left(1+\frac{4}{y}\right)^{1 / 2}\right]=\nu_{1}(y) .
$$

In this case, the transition function $\zeta(y)$ provided by the "graviton picture" is identical to the MOND transition function $\nu_{n}(y)$ for $n=1$. I illustrate the functions $\xi^{-1}(x)=\mu_{1}^{-1}(x)$ and $\zeta(y)=\nu_{1}(y)$ in Fig. 1 ; the diagrams should be compared to Fig. 10 of Famaey \& McGaugh (2012) and Fig. 11 of Kroupa (2012).

\section{DISCUSSION}

"It is principally the elegance of general relativ-
ity theory and its success in solar system tests
that lead us to the bold extrapolation that the
gravitational acceleration has the form $G M / r^{2}$
on scales $10^{21}-10^{26} \mathrm{~cm}$ that are relevant for the
solar neighborhood, galaxies, clusters of galax-
ies, and superclusters."
- Binney \& Tremaine (1987), Ch. 10.4 , p. 635

Historically, the missing mass problem has usually been approached by postulating non-luminous and 

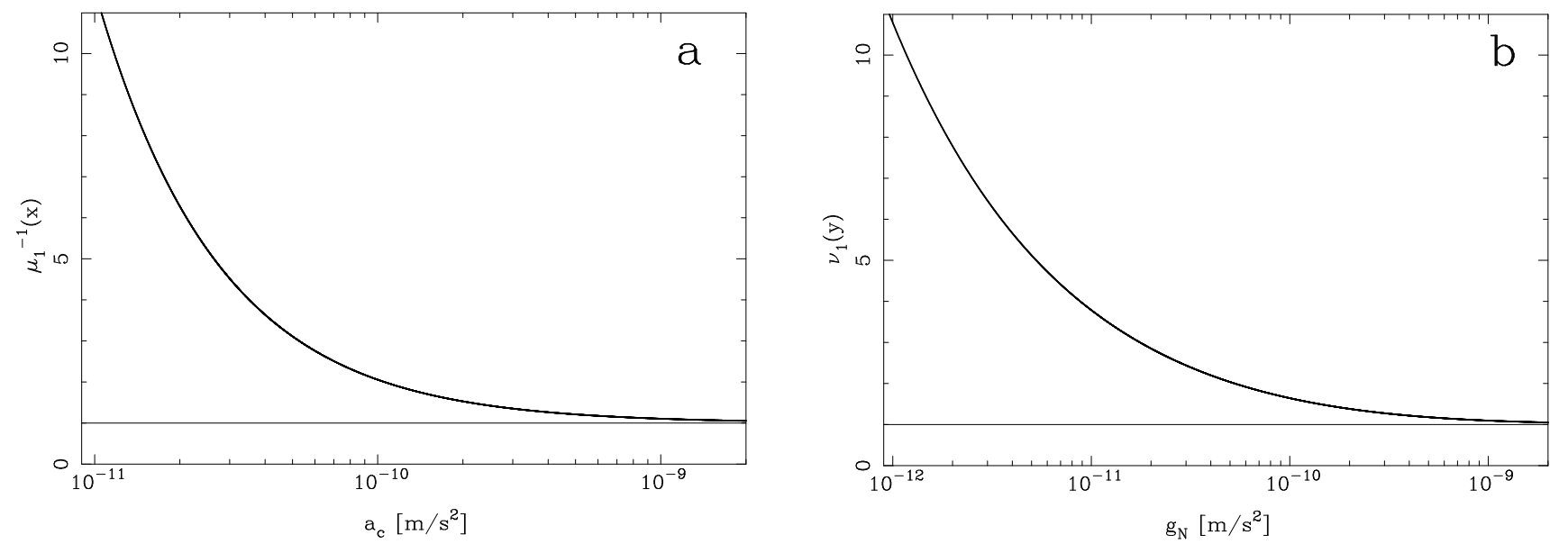

Fig. 1. - The transition functions derived from comparison of MOND and "graviton picture", assuming $a_{\mathrm{M}}=1.1 \times$ $10^{-10} \mathrm{~m} \mathrm{~s}^{-2}$. a. The inverse of the function $\mu_{1}(x)$ vs. $a_{\mathrm{c}}$. b. The function $\nu_{1}(y)$ vs. $g_{\mathrm{N}}$. Please note the logarithmic-linear axis scales. These diagrams should be compared to Fig. 10 of Famaey \& McGaugh (2012) and Fig. 11 of Kroupa (2012).

non-baryonic dark matter (Ostriker \& Peebles 1973; Einasto et al. 1974), eventually evolving into the $\Lambda$ CDM standard model of cosmology (e.g., Bahcall et al. 1999). In recent years, it has become clear that this approach is incomplete. The assumption of dark matter distributed within and around galaxies is partially incompatible with observations of structure and dynamics of galaxies and groups of galaxies (see Kroupa 2012 for a recent review). Standard cosmology seems unable to predict fundamental relations of galactic dynamics like the Faber-Jackson and Tully-Fisher relations (Faber \& Jackson 1976; Tully \& Fisher 1977), the MDA relation, or the surface brightness-acceleration relation. More generally, it has been found that galactic dynamics is intimately linked with a universal characteristic acceleration which can be identified with Milgrom's constant $a_{\mathrm{M}} \approx 10^{-10} \mathrm{~m} \mathrm{~s}^{-2}$ (e.g., Famaey \& McGaugh 2012).

The dynamics of galaxies and groups of galaxies can be understood in the frame of theories of modified gravity and/or inertia based on acceleration scales - a discovery eventually leading to MOND (e.g., Milgrom 1983a; Bekenstein \& Milgrom 1984; Sanders \& McGaugh 2002; Ferreira \& Starkman 2009). On the one hand, MOND has been remarkably successful in describing galactic dynamics while circumventing the problems that plague $\Lambda$ CDM cosmology (cf. Section 1; also Ferreira \& Starkman 2009; Famaey \& McGaugh 2012; Kroupa 2012). On the other hand, MOND is obviously incomplete: it does not derive from a physical mechanism but rather empirically from the boundary conditions provided by observations. In addition, it does not comprise a prediction for the transitional regime from Newtonian to modified dynamics; appropriate scaling laws have to be constructed from comparison to observations.
The "graviton picture" of gravitation (Trippe 2013) starts off from a physical mechanism: the (ad-hoc) assumption that gravity is mediated by virtual massive gravitons that obey certain rules of interaction. From this it is possible to derive the scaling law given by Eq. 5 which is in good agreement with observations. As shown in Section 2.2, this scaling law actually comprises the empirical scaling laws employed in the frame of MOND - at least for non-relativistic dynamical systems and circular orbit geometries.

The "graviton picture" as well as MOND each comprise a characteristic acceleration, either $a_{\mathrm{M}}$ or $a_{0}$, with $a_{\mathrm{M}}=8 \pi a_{0}$. These characteristic accelerations are free parameters. Empirically, $a_{\mathrm{M}} \approx c H_{0} / 2 \pi$ with $c$ denoting the speed of light and $H_{0}$ denoting Hubble's constant (e.g., Famaey \& McGaugh 2012). This might suggest a physical connection between galactic dynamics and cosmology; however, as yet this relation is entirely empirical and has not been derived from first principles.

Evidently, the new feature I add to the discussion of MONDian scaling laws of gravitation is the theoretical MDA relation (Eq. 5). This relation implies a proportionality of total dynamical mass $M_{\text {tot }}$ and luminous baryonic mass $M_{0}$ - a property expected for modified laws of gravity but sharply distinct from standard dark matter models where dark and luminous mass are distributed independently. Such a tight relation between luminous and baryonic mass is observed in galactic rotation curves (known as "Renzo's rule"; Sancisi 2004) and in the "Train Wreck" (A520) cluster of galaxies (Jee et al. 2012). A possible counter-example is provided by the "Bullet Cluster" of galaxies (1E0657-56); here observations indicate a spatial separation of dark and luminous mass but also find the cluster kinematics to be inconsistent with $\Lambda$ CDM cosmology (Lee \& Komatsu 2010) - making the overall analysis of the 
"Bullet Cluster" rather inconclusive actually. A more detailed discussion of various observational tests of the "graviton picture" is provided in Trippe (2013).

Regarding the combined evidence, I conclude that (classical) MOND naturally derives from the "graviton picture". Eventually, this suggests that the "graviton picture" - despite its toy-model character (Trippe 2013) - provides a valid candidate for the physical mechanism behind MOND and gravity on galactic scales in general - at least to first order.

\section{CONCLUSIONS}

I compare Modified Newtonian Dynamics with the "graviton picture" of gravitation which I proposed recently, and arrive at the following principal conclusions:

1. MOND and the "graviton picture" find identical expressions for dynamics in the limits of strong and weak centripetal accelerations. Their parameters, $a_{\mathrm{M}}$ and $a_{0}$ respectively, are related as $a_{\mathrm{M}} \equiv 8 \pi a_{0}$; empirically, $a_{\mathrm{M}} \approx 10^{-10} \mathrm{~m} \mathrm{~s}^{-2}$.

2. The empirical scaling laws commonly used in MOND, which are needed to quantify the transitional regime between Newtonian and modified dynamics, are contained in the "graviton picture" a priori.

3. MOND derives from the "graviton picture" - at least for non-relativistic, highly symmetric dynamical systems.

These conclusions suggest that - at least to first order - the "graviton picture" provides a valid candidate for the physical mechanism behind MOND and gravity on galactic scales in general.

\section{ACKNOWLEDGMENTS}

I am grateful to JunghwAN OH, TAESEOK LEE, JAE-Young KIM, and Jong-Ho PARK (all at SNU) for valuable technical support. This work made use of the software package DPUSER ${ }^{\dagger}$ developed and maintained by THOMAs OTT at MPE Garching. I acknowledge financial support from the Korean National Research Foundation (NRF) via Basic Research Grant 2012R1A1A2041387. Last but not least, I am grateful to an anonymous referee whose comments helped to improve the quality of this paper.

\section{REFERENCES}

Bahcall, N. A., Ostriker, J. P., Perlmutter, S., \& Steinhardt, P. J. 1999, The Cosmic Triangle: Revisiting the State of the Universe, Science, 284, 1481

Bekenstein, J., \& Milgrom, M. 1984, Does the Missing Mass Problem Signal the Breakdown of Newtonian Gravity?, ApJ, 286, 7

\footnotetext{
${ }^{\dagger}$ http: //www.mpe.mpg.de/ ott/dpuser/dpuser.html
}

Bekenstein, J. 2006, The Modified Newtonian Dynamics MOND and Its Implications for New Physics, Contemp. Phys., 47, 387

Binney, J., \& Tremaine, S. 1987, Galactic Dynamics (Princeton: Princeton University Press)

Einasto, J., Kaasik, A., \& Saar, E. 1974, Dynamic Evidence on Massive Coronas of Galaxies, Nature, 250, 309

Faber, S. M., \& Jackson, R. E. 1976, Velocity Dispersions and Mass-to-Light Ratios for Elliptical Galaxies, ApJ, 204, 668

Famaey, B., \& Binney, J. 2005, Modified Newtonian Dynamics in the Milky Way, MNRAS, 363, 603

Famaey, B., \& McGaugh, S. S. 2012, Modified Newtonian Dynamics (MOND): Observational Phenomenology and Relativistic Extensions, Living Rev. Relativity, 15, 10

Ferreira, P. G., \& Starkman, G. D. 2009, Einstein's Theory of Gravity and the Problem of Missing Mass, Science, 326,812

Jee, M. J., Mahdavi, A., Hoekstra, H., et al. 2012, A Study of the Dark Core in A520 with the Hubble Space Telescope: The Mystery Deepens, ApJ, 747, 96

Kroupa, P. 2012, The Dark Matter Crisis: Falsification of the Current Standard Model of Cosmology, PASA, 29, 395

Lee, J., \& Komatsu, E. 2010, Bullet Cluster: a Challenge to $\Lambda$ CDM Cosmology, ApJ, 718, 60

McGaugh, S. S. 2004, The Mass Discrepancy - Acceleration Relation: Disk Mass and the Dark Matter Distribution, ApJ, 609, 652

Milgrom, M. 1983, A Modification of the Newtonian Dynamics as a Possible Alternative to the Hidden Mass Hypothesis, ApJ, 270, 365

Milgrom, M. 1983, A Modification of the Newtonian Dynamics: Implications for Galaxies, ApJ, 270, 371

Milgrom, M. 1983, A Modification of the Newtonian Dynamics: Implications for Galaxy Systems, ApJ, 270, 384

Ostriker, J. P., \& Peebles, P. J. E. 1973, A Numerical Study of the Stability of Flattened Galaxies: Or, Can Cold Galaxies Survive?, ApJ, 186, 467

Rubin, U. C., Ford, W. K. Jr., \& Thonnard, N. 1980, Rotational Properties of 21 Sc Galaxies with a Large Range of Luminosities and Radii, from NGC $4605(\mathrm{R}=4 \mathrm{kpc})$ to UGC 2885 ( $\mathrm{R}=122 \mathrm{kpc})$, ApJ, 238, 471

Sancisi, R. 2004, The Visible Matter - Dark Matter Coupling, in: Ryder, S. D., et al. (eds.), Dark Matter in Galaxies, IAU Symposium, 220, 233

Sanders, R. H. 1990, Mass Discrepancies in Galaxies: Dark Matter and Alternatives, A\&AR, 2, 1

Sanders, R. H., \& McGaugh, S. S. 2002, Modified Newtonian Dynamics as an Alternative to Dark Matter, ARA\&A, 40, 263

Trippe, S. 2013, A Simplified Treatment of Gravitational Interaction on Galactic Scales, JKAS, 46, 41

Tully, R. B., \& Fisher, J. R. 1977, A New Method of Determining Distances to Galaxies, A\&A, 54, 661

Zwicky, F. 1933, Die Rotverschiebung von Extragalaktischen Nebeln, Helv. Phys. Acta, 6, 110 